\title{
Estudios colaborativos multicéntricos en cirugía: ¿qué sucede en Latinoamérica?
}

\author{
Surgical multicenter collaborative studies: ¿What happen in Latin America?
}

Luis A. Boccalatte ${ }^{1,2 *}$, Ma. Marta Modolo ${ }^{3,4}$, José A. Calvache ${ }^{5,6}$, Eddy P. Lincango-Naranjo7, Gustavo M. Ataíde-Gomes ${ }^{8}$, Igor Lima-Buarque ${ }^{8}$, Carlos J. Pérez-Rivera ${ }^{9,10}$, Sebastián B. Shu-Yip ${ }^{11}$, Ximena P. Vásquez-Ojeda" ${ }^{11}$, Maricarmen Olivos ${ }^{5}$, Sylvia Batista ${ }^{12}$, Ma. Lorena Aguilera-Arévalo ${ }^{13}$, Hugo Gómez-Fernández ${ }^{14}$, Danilo Arévalo ${ }^{15}$, Dimitri Nepogodievv' ${ }^{16}$, Maria Picciochil y Joana Simoes ${ }^{18}$ ${ }^{1}$ Servicio de Cirugía General, Hospital Italiano de Buenos Aires, Buenos Aires, Argentina; ${ }^{2}$ Departamento de Cirugía, Instituto Universitario Hospital Italiano, Buenos Aires, Argentina; ${ }^{3}$ Servicio de Cirugía General, Hospital Barros Luco Trudeau, Región Metropolitana de Santiago, Chile; ${ }^{4}$ Servicio de Cirugía General, Hospital Luis Lagomaggiore, Mendoza, Argentina; ${ }^{5}$ Servicio de Cirugía, Hospital de Niños Roberto del Río, Universidad de Chile, Santiago, Chile; ${ }^{6}$ Departamento de Anestesiología, Erasmus University Medical Center, Rotterdam, Países Bajos; ${ }^{7}$ Unidad de Investigación de Conocimientos y Evaluación, Mayo Clinic, Rochester, Minnesota, Estados Unidos; ${ }^{8}$ Faculdad de Medicina, Centro Universitário CESMAC, Maceió, Brasil; ${ }^{\circ}$ Departamento de Cirugía General, Fundación Cardioinfantil-Instituto de Cardiología, Bogotá, Colombia; ${ }^{10}$ Departamento de Cirugía General, Universidad El Bosque, Bogotá, Colombia; ${ }^{11}$ Universidad Peruana Cayetano Heredia, Lima, Perú; ${ }^{12}$ Departamento de Cirugía y Especialidad, INTEC (Instituto Tecnológico de Santo Domingo), Santo Domingo, República Dominicana; ${ }^{13}$ Departamento de Cirugía, Hospital General San Juan de Dios, Universidad de San Carlos de Guatemala, Guatemala; ${ }^{14}$ Diseminación y Coordinación Global Surg, Asunción, Paraguay; ${ }^{15}$ Centro Médico de la Mujer ARCO, San Salvador, El Salvador; ${ }^{16} \mathrm{NIHR}$ Unit on Global Surgery, University of Birmingham, Birmingham, Reino Unido; ${ }^{17}$ America's Coordinator GlobalSurg-CovidSurg Week, North America, Central America, South America, Caribbean, Hospital Professor Doutor Fernando Fonseca, Amadora, Portugal

\section{Resumen}

Antecedentes: GlobalSurg es un grupo internacional de investigadores que tiene como propósito la conducción y la diseminación de robustos estudios colaborativos, internacionales y multicéntricos. Objetivo: Exponer las estrategias necesarias y las barreras encontradas en la conducción de estudios multicéntricos masivos en cirugía. Método: Durante el segundo semestre del año 2020 se llevó a cabo el estudio Surg-Week Prospective International Cohort Study, hasta la fecha el estudio internacional más grande en el campo de la cirugía, con 141,582 pacientes incluidos. Un total de 4975 miniequipos, de uno a cinco integrantes, recopilaron datos de 116 países de todos los continentes. Resultados: La creación de un sitio web oficial del estudio, reportes con información relevante vía e-mail o grupos vía WhatsApp, conformación de un comité de diseminación del protocolo, dictado de webinars sobre publicaciones recientes del equipo, designación de líderes nacionales e internacionales, y la divulgación por medio de sociedades, fueron las estrategias utilizadas para el desarrollo de la investigación. Sin embargo, las barreras detectadas para llevar a cabo el estudio multicéntrico fueron variadas. Conclusiones: Los trabajos colaborativos permiten establecer redes entre diferentes profesionales con el fin de mejorar la calidad de la gestión, las políticas sanitarias y la atención a los pacientes en tiempos de constante cambio.

Palabras clave: Investigación. Cirugía. Científicos. Investigadores. Barreras. Coordinación. 


\begin{abstract}
Background: GlobalSurg is an international group of researchers whose purpose is to conduct and disseminate robust collaborative, international and multicenter studies. Objective: To expose the necessary strategies and the barriers crossed in conducting massive multicenter studies in surgery. Method: During the second semester of 2020, the study Surg-Week Prospective International Cohort Study was carried out. Surg-Week has been the largest international study in the field of surgery to date, with 141,582 patients included. A total of 4975 mini-teams, of between 1 and 5 members, collected data from 116 countries on all continents. Results: The creation of an official website for the study, reports with relevant information via email or groups via WhatsApp, formation of a Dissemination Committee of the protocol, delivery of webinars on recent team publications, appointment of leaders at the national and international level, and outreach through partnerships, were the strategies used for the development of the research. However, the barriers turned out to involve different aspects. Conclusions: Collaborative work allows establishing networks between different professionals with the goal of improving the quality of management, health policies and care of our patients in a timely manner of constant change.
\end{abstract}

Key words: Research. Surgery. Scientifics. Researchers. Barriers. Coordination.

\section{Introducción}

GlobalSurg es un grupo internacional de investigadores en numerosas áreas con interés en la medicina perioperatoria que tiene como propósito la conducción y la diseminación de robustos estudios prospectivos, observacionales y aleatorizados, colaborativos, internacionales y multicéntricos, con impacto en la práctica quirúrgica diaria, coordinados por el National Institute for Health Research (NIHR), Global Health Research Unit on Global Surgery, en el Reino Unido. Dicha colaboración está en directa consonancia con la gran necesidad de integrar el conocimiento para el mejoramiento científico actual' ${ }^{1}$. Esta red de investigadores se extendió poco después de determinar el estado de pandemia, como respuesta a la necesidad de responder interrogantes relacionados con el SARS-CoV-2 en el ámbito quirúrgico. Por esta razón surgió el CovidSurg Collaborative.

En el segundo semestre del año 2020 se llevó a cabo el estudio Surg-Week Prospective International Cohort Study, el estudio internacional más grande en el campo de la cirugía hasta la fecha, con 141,582 pacientes incluidos. Un total de 4975 miniequipos, de entre uno y cinco integrantes, recopilaron datos en 116 países de todos los continentes. Es el primer estudio panespecialidad que también incluye niños $(9,7 \%$ del total de los pacientes incluidos, $n=13,800)$. Hubo una gran rigurosidad en lo que respecta a criterios de inclusión y exclusión, y de carga de datos en RedCap, siendo la tasa de datos faltantes para los campos de datos clave de solo el $0,2 \%$.
RedCap es un sistema seguro de acceso online para la recolección de datos anónimos de pacientes. Es accesible desde cualquier lugar del mundo, lo que posibilita la conectividad de los equipos de investigación y logra recopilar datos de alta calidad $^{2}$.

\section{Método}

El objetivo primario del estudio fue determinar el momento óptimo de la cirugía electiva durante la pandemia de COVID-19 y, en segundo lugar, determinar indicadores quirúrgicos globales relevantes, como la tasa de mortalidad posoperatoria a los 30 días y las complicaciones asociadas (complicaciones pulmonares, tromboembolia pulmonar y complicaciones según la clasificación de Dindo-Clavien). Se utilizaron tres cohortes de pacientes: 1) los que padecieron la infección antes de la cirugía, 2) los que se infectaron durante el posoperatorio y 3) aquellos sin infección preoperatoria ni posoperatoria. El estudio fue dirigido a todos los niveles hospitalarios (con diversos grados de complejidad), especialidades quirúrgicas, grupos de edad y tipos de cirugía (ambulatoria, internación, programada y urgencia). Se recolectaron datos por un periodo de 7 días (4 semanas del mes de octubre) y tuvo un seguimiento posoperatorio de 30 días. Una publicación preliminar del mismo grupo describió que las complicaciones pulmonares y la mortalidad a 30 días cae al $0 \%$ cuando la cirugía es diferida 4 semanas desde la positividad del test para SARS-CoV-2 ${ }^{3}$. Más detalles acerca del estudio pueden encontrarse en su sitio web (https://globalsurg.org/surgweek/). 
Tabla 1. Distribución de equipos y pacientes enrolados en América Latina

\begin{tabular}{|c|c|c|c|c|c|}
\hline $\begin{array}{l}\text { Países } \\
(n=16)\end{array}$ & $\begin{array}{l}\text { Hospitales } \\
(n=152)\end{array}$ & $\begin{array}{l}\text { Mini equipos } \\
\quad(n=377)\end{array}$ & $\begin{array}{l}\text { Pacientes enrolados } \\
\qquad(n=11,168)\end{array}$ & Pacientes enrolados por miniequipo & Pacientes enrolados por hospital \\
\hline Argentina & 9 & 46 & 1911 & 41.54 & 212.33 \\
\hline Barbados & 1 & 3 & 13 & 4.33 & 13 \\
\hline Brasil & 39 & 70 & 1906 & 27.23 & 48.87 \\
\hline Chile & 9 & 15 & 459 & 30.60 & 51 \\
\hline Colombia & 22 & 78 & 2605 & 33.40 & 118.41 \\
\hline Cuba & 1 & 1 & 2 & 2 & 2 \\
\hline $\begin{array}{l}\text { República } \\
\text { Dominicana }\end{array}$ & 2 & 33 & 399 & 12.09 & 199.50 \\
\hline Ecuador & 2 & 2 & 54 & 27 & 27 \\
\hline El Salvador & 1 & 1 & 11 & 11 & 11 \\
\hline Guatemala & 10 & 16 & 567 & 35.44 & 56.70 \\
\hline México & 26 & 44 & 1406 & 31.95 & 54.08 \\
\hline Panamá & 1 & 1 & 10 & 10 & 10 \\
\hline Paraguay & 11 & 39 & 988 & 25.33 & 89.82 \\
\hline Perú & 15 & 22 & 698 & 31.73 & 46.53 \\
\hline $\begin{array}{l}\text { Trinidad y } \\
\text { Tobago }\end{array}$ & 1 & 2 & 32 & 16 & 32 \\
\hline Uruguay & 2 & 4 & 107 & 26.75 & 53.50 \\
\hline
\end{tabular}

\section{Resultados}

\section{Participación de América Latina}

En América Latina participaron 377 miniequipos de 16 países, reclutando un total de 11,168 pacientes. De hecho, en América Latina no habíamos visto nunca una colaboración tan exitosa en el ámbito quirúrgico. Los países participantes (Fig. 1), el número de equipos por país y la cantidad de pacientes enrolados se detallan en la tabla 1. El número de centros y de países que participaron en el estudio SurgWeek fue mayor que en los estudios realizados en años anteriores por GlobalSurg Collaborative. Este equipo internacional busca guiar la práctica clínica basándose en la evidencia generada de alta calidad, centrándose en los resultados de los pacientes y apuntando a cambiar y orientar las políticas sanitarias según el nivel institucional y los recursos, siempre contextualizando los resultados obtenidos a países de similar desarrollo humano.

Finalmente, la participación latinoamericana en esta colaboración ha sido sustancial, siendo Colombia y

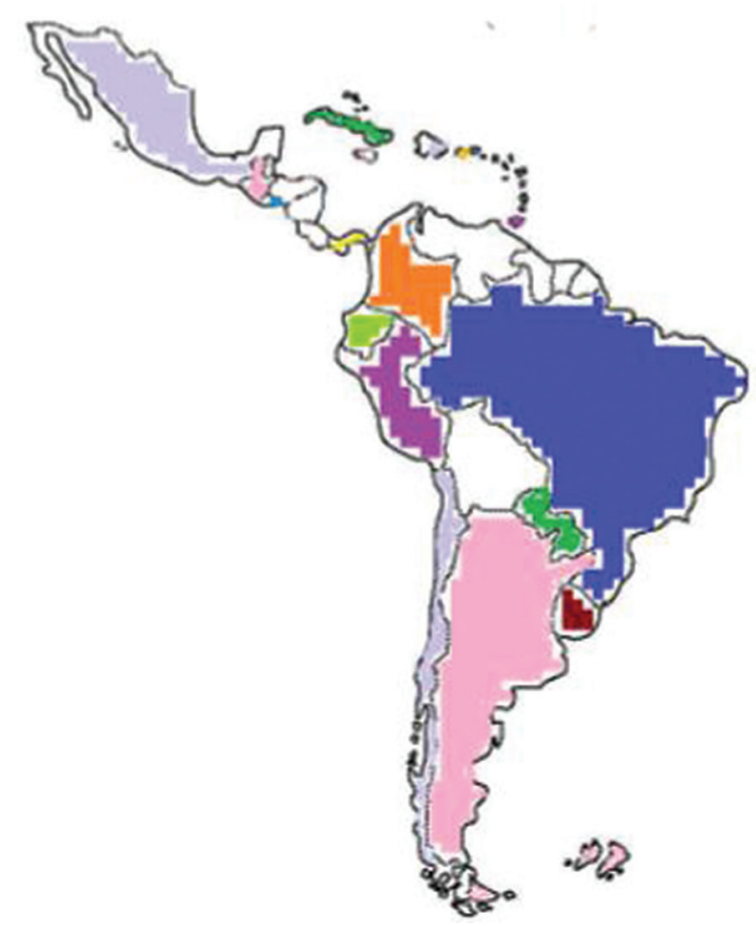

Figura 1. Países participantes de América Latina: Colombia, Argentina, Brasil, México, Paraguay, Perú, Guatemala, Chile, República Dominicana, Uruguay, Ecuador, Trinidad y Tobago, Puerto Rico, El Salvador, Panamá y Cuba. 
Tabla 2. Estrategias utilizadas para la coordinación del estudio, ventajas y barreras impuestas por el método elegido

\begin{tabular}{|c|c|c|c|}
\hline Estrategia de coordinación & Objetivo & Ventajas & Barreras y aspectos de mejora \\
\hline Sitio web & $\begin{array}{l}\text { Agrupamiento de la información en un sitio } \\
\text { online accesible y traducido a diferentes } \\
\text { idiomas } \\
\text { Ejemplos: } \\
\text { Protocolo de investigación } \\
\text { Material audiovisual explicativo } \\
\text { Modelos de aprobación del comité de ética y } \\
\text { consentimiento informado }\end{array}$ & $\begin{array}{l}\text { Disponibilidad de recursos } \\
\text { útiles y accesibles } \\
\text { Forma rápida de } \\
\text { introducción al modelo y al } \\
\text { objetivo del estudio para } \\
\text { hospitales interesados }\end{array}$ & $\begin{array}{l}\text { Secesidad de conexión a internet } \\
\text { estable } \\
\text { La traducción literal del protocolo } \\
\text { a distintos idiomas puede generar } \\
\text { confusión en los investigadores } \\
\text { interesados }\end{array}$ \\
\hline E-mail & $\begin{array}{l}\text { Reclutamiento de investigadores: distribución } \\
\text { global según bases de datos previas de } \\
\text { profesionales que ya habían participado en } \\
\text { trabajos del consorcio colaborativo } \\
\text { Coordinación general (masiva) del desarrollo } \\
\text { de la investigación } \\
\text { Ejemplos: } \\
\text { Comienzo del estudio } \\
\text { Fechas límite } \\
\text { Aclaraciones } \\
\text { Autorías } \\
\text { Publicaciones }\end{array}$ & $\begin{array}{l}\text { Aumento de la } \\
\text { incorporación de nuevos } \\
\text { centros } \\
\text { Otorgó fluidez del estudio a } \\
\text { través de la guía continua } \\
\text { de los investigadores }\end{array}$ & $\begin{array}{l}\text { Algunos avisos correspondían a } \\
\text { publicaciones previas que llevaban a } \\
\text { la confusión de los investigadores no } \\
\text { arelacionados con ellas } \\
\text { Excesivo número y contenido } \\
\text { de correos (en determinados } \\
\text { momentos) }\end{array}$ \\
\hline Grupos de Whatsapp & $\begin{array}{l}\text { Difusión de los puntos expresados } \\
\text { previamente en «Sitio web» } \\
\text { Regionalización de los } \\
\text { investigadores (América, Europa, Asia, } \\
\text { Dissemination Committee, Help Desk support, } \\
\text { Hospital leads, etc.) } \\
\text { Grupos nacionales con difusión de } \\
\text { información traducida }\end{array}$ & $\begin{array}{l}\text { Comunicación más fluida } \\
\text { Resolución más rápida de } \\
\text { los problemas de cada } \\
\text { investigador } \\
\text { t, Aumento de la } \\
\text { participación y de puntos } \\
\text { de vista } \\
\text { Discusión de aspectos } \\
\text { comunes a una región }\end{array}$ & $\begin{array}{l}\text { Exceso de mensajes } \\
\text { Mensajes recibidos en diferentes } \\
\text { husos horarios } \\
\text { Necesidad de buen dominio } \\
\text { del idioma inglés escrito, salvo } \\
\text { excepciones }\end{array}$ \\
\hline Comité de diseminación & $\begin{array}{l}\text { Transmitir los principales lineamientos } \\
\text { del estudio y actualizaciones a cada líder } \\
\text { nacional }\end{array}$ & $\begin{array}{l}\text { Identificación de un } \\
\text { responsable por país para } \\
\text { efectivizar las normativas }\end{array}$ & $\begin{array}{l}\text { Debido al gran número de } \\
\text { integraciones, la interacción } \\
\text { individualizada es difícil } \\
\text { Necesidad de buen dominio del } \\
\text { idioma inglés }\end{array}$ \\
\hline Webinar & $\begin{array}{l}\text { Informar acerca de actualizaciones y puesta } \\
\text { al día del desarrollo del estudio en los } \\
\text { diferentes países }\end{array}$ & $\begin{array}{l}\text { Suplir las necesidades e } \\
\text { inquietudes de cada país }\end{array}$ & $\begin{array}{l}\text { Debido a las diferencias horarias, } \\
\text { muchos asistentes no pudieron } \\
\text { conectarse en tiempo y forma }\end{array}$ \\
\hline Líder nacional y hospitalario & $\begin{array}{l}\text { Asegurar que el estudio se lleve a cabo } \\
\text { en ese país y hospital, y recurrir a él si se } \\
\text { identifican problemas }\end{array}$ & $\begin{array}{l}\text { Organización y } \\
\text { cumplimiento del estudio } \\
\text { Posición de liderazgo y } \\
\text { manejo de grupo (etapa de } \\
\text { crecimiento personal) }\end{array}$ & $\begin{array}{l}\text { El rol implica mucho compromiso } \\
\text { y dedicación, por lo que algunos } \\
\text { países no contaban con líder } \\
\text { enacional } \\
\text { Manejo fluido del idioma inglés }\end{array}$ \\
\hline $\begin{array}{l}\text { Sociedades científicas } \\
\text { locales }\end{array}$ & $\begin{array}{l}\text { Difusión del estudio en diversos formatos (4) } \\
\text { Fomentar el enrolamiento de cirujanos y } \\
\text { residentes de cirugía en investigación }\end{array}$ & $\begin{array}{l}\text { Las sociedades científicas } \\
\text { gozan de reconocimiento } \\
\text { nacional y captan la } \\
\text { atención de sus miembros } \\
\text { Fortalecer una red de } \\
\text { contactos entre cirujanos o } \\
\text { participantes }\end{array}$ & $\begin{array}{l}\text { Los no miembros no accedieron a la } \\
\text { información } \\
\text { Algunas sociedades científicas no } \\
\text { apoyaron la divulgación del estudio } \\
\text { Baja tasa de respuestas }\end{array}$ \\
\hline
\end{tabular}

Argentina los países que más pacientes aportaron. En la región, estos países contribuyeron con un $42 \%$ del total, siendo sus capitales las que aportaron más pacientes reclutados y seguidos a 30 días.
Adicionalmente, la República Argentina fue el país que presentó mayor ratio de número de pacientes por miniequipo, con 41.54 (nueve centros registrados en el país). Cabe señalar que 181 miniequipos presentaron 
Tabla 3. Barreras observadas durante el desarrollo del estudio

\begin{tabular}{ll}
\hline $\begin{array}{l}\text { Barreras en países de } \\
\text { América Latina }\end{array}$ & Posibles causas \\
\hline Idiomáticas & $\begin{array}{l}\text { Español como idioma nativo en la mayoría de los países } \\
\text { La población médica no cuenta, mayormente, con altos } \\
\text { niveles de inglés }\end{array}$
\end{tabular}

Estrategias de mejora
Fomentar y formar a los médicos durante estancias formativas tempranas (prácticas hospitalarias, residencias, etc.) en inglés
Formación en una segunda lengua de forma activa en las universidades
A corto plazo: generar traducciones del protocolo principal y documentación necesaria

Condiciones del sistema de salud

Registro inadecuado de datos en la historia clínica pandemia

Registros quirúrgicos incompletos en el área de síntomas de COVID-19 por no pertenecer a la especialidad

Problemas para obtener la aprobación del estudio por el comité de ética o la autorización ministerial

Competencia y egoísmo en diferentes estratos debilitados, cuyos recursos humanos no eran suficientes debido a la demanda de especialistas del campo quirúrgico, como los anestesiólogos

Alto número del personal de salud enfermo

Poca disponibilidad y lentitud de resultado de pruebas de reacción en cadena de la polimerasa y acceso a imágenes de tomografía computada

Escasa disponibilidad de insumos médicos y quirúrgicos en países donde la importación no estuvo disponible Algunos centros (privados mayoritariamente) pudieron responder estratégicamente y con efectividad a las condiciones adversas derivadas de la pandemia Debilidad estructural

Ejemplo:

Menor cantidad de camas de cuidados intensivos para los cuidados posoperatorios, cancelación completa de las cirugías electivas, etc.

Países que no cuentan con historia clínica informatizada mposibilidad o menor seguimiento de los pacientes por la

Comités de ética cerrados o muy lentos en su resolución Comités de ética con poca formación en este tipo de estudios (solicitud de papeles innecesarios)

Falta de personal

Ministerio de salud sin conocimientos de la realización de estudios internacionales

Falta de aprobación por investigadores novatos o inexpertos

Necesidad de protagonismo y reconocimiento individual y colectivo
Impacto de la pandemia en los sistemas de salud crónicamente Fortalecimiento de los sistemas de salud a través

Comprender el concepto de trabajo colaborativo y sus ventajas Aumentar el número de publicaciones indexadas

Falta de liderazgo de políticas coherentes

Horario protegido para la actividad científica Distribución equitativa de los recursos sanitarios y de los recursos para investigación Fomentar la investigación en residentes/becados Establecer protocolos institucionales según la disponibilidad de recursos humanos, estructurales y económicos

Historia clínica universal preformada y digitalizada a través de un servidor con acceso encriptado Capacitar en la importancia de la correcta y completa documentación, el seguimiento y el registro de los datos en estudios de investigación Sistema de codificación universal CIE-10 para el registro de diagnósticos y procedimientos

Estimular la creación de comités de ética Capacitar a los comités sobre este tipo de estudios Agilidad de las resoluciones mediante sesiones virtuales

Conocimiento de no solicitar consentimiento informado en estudios observacionales

Formación metodológica extendida en comités hospitalarios y de investigación

Enaltecer en pregrado y sistema de residencias médicas la importancia de la investigación científica y el trabajo colaborativo entre colegas con el fin de mejorar la calidad de atención de nuestros pacientes

Incredulidad de que el estudio pudiera ser internacionalmenteComprensión de la actividad médica y sanitaria reconocido

Falta de incentivo económico y de rédito profesional Falta de comunicación y ayuda colectiva entre pares de la misma institución, región o provincia como ciencia Incentivar el trabajo en equipo y mejorar la comunicación entre colegas Incentivar la investigación en el campo quirúrgico Falta de identidad o compromiso institucional, regional y nacional a tiempo protegido y remunerado a través de Poco tiempo disponible para que los cirujanos se dediquen a colaboraciones internacionales y nacionales con la investigación las sociedades científicas

Acceso a RedCap por medio de subsidios a instituciones con alta producción científica Acceso a la plataforma RedCap proporcionado por el estudio para introducir los datos recopilados de los pacientes de cada institución

En trabajos colaborativos como este, el acceso al sistema es gratuito para los colaboradores 


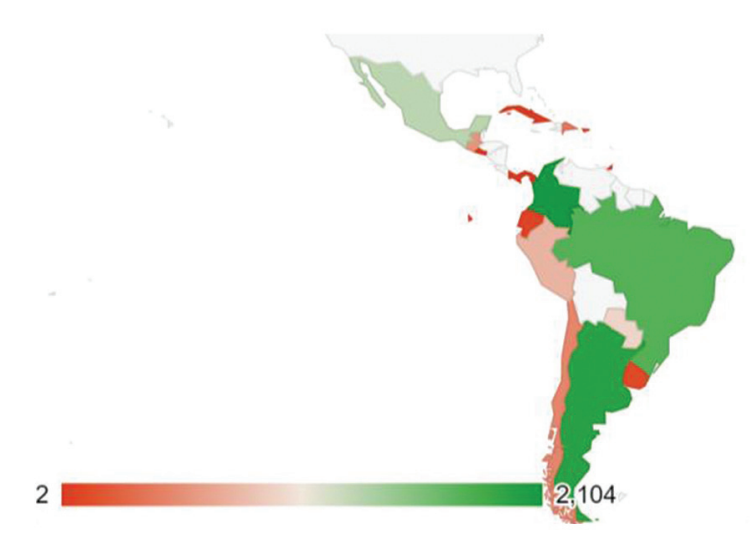

Figura 2. Número de pacientes reclutados en cada país.

menos de 20 pacientes, y las razones pueden ser varias: 1) los centros más pequeños del interior del país disminuyeron sustancialmente la actividad quirúrgica; 2) la escasez o falta de datos para completar la totalidad del formulario o pocos pacientes reclutados; 3 ) agotamiento físico y mental del equipo o imposibilidad de seguimiento de los pacientes en el tiempo; y 4) disminución del número de cirugías por no contar con una sala de operaciones exclusiva para pacientes contagiados por coronavirus.

La figura 2 muestra el grado de reclutamiento de los países participantes.

\section{Estrategias de coordinación de estudios multicéntricos}

Este grupo presenta diferentes principios relevantes de investigación colaborativa. Dentro de su conformación se encuentra un equipo central responsable de la coordinación del proyecto completo, con base en la Universidad de Birmingham, Reino unido. Para la diseminación y la promoción del enrolamiento de la mayor cantidad de centros posible se creó un comité de diseminación, que incluye líderes nacionales responsables de coordinar el estudio en los diferentes países participantes. En la tabla 2 se exponen las estrategias utilizadas para la coordinación del estudio, la resolución de dudas y la emisión de recomendaciones, señalando a su vez los aciertos y las barreras que se encontraron.

\section{Barreras en el desarrollo de estudios multicéntricos en América Latina}

Nuestro mayor interés es mejorar la calidad de la atención de los pacientes. Sin embargo, la posibilidad de encontrarse citados en revistas internacionales de alto impacto e indexadas resulta atractiva para todos los colaboradores vinculados. A través de nuestro rol como líderes nacionales se pudieron identificar obstáculos o dificultades tanto en la coordinación como en el desarrollo y la conducción del proyecto ${ }^{4}$. Una de las primeras apreciaciones fue la diferencia temporal en la diseminación y el curso de la pandemia y el desarrollo del estudio entre Europa y América Latina, lo que implicó muchos recursos humanos dedicados a la actividad asistencial, en detrimento de la actividad académica e investigativa, en diferentes periodos de tiempo. Además, la deficiencia de recursos socioeconómicos de las naciones latinoamericanas comparadas con las europeas acrecentó esta brecha, implicando una gran cantidad de investigadores abocados exclusivamente a la tarea asistencial. Por esta razón, fue necesario duplicar el esfuerzo para lograr un mayor reclutamiento y enrolamiento de centros manteniendo el interés y la calidad del estudio. Otro punto crítico al hablar de barreras fue la aprobación de los comités de ética institucional locales, ministeriales o nacionales. La mayoría de las solicitudes resultaron difíciles por los motivos antedichos, y en otros centros exigieron documentación innecesaria. El exceso de burocracia y la poca facilidad para incorporar procesos digitales llevó a los investigadores a desistir en el intento de participación. Otros inconvenientes que se presentaron durante la puesta en marcha y el reclutamiento de los pacientes se detallan en la tabla 3.

\section{Conclusiones}

La puesta a punto y la ejecución de estudios multicéntricos colaborativos es una estrategia fundamental y necesaria para obtener estudios de alta calidad científica y buen nivel de evidencia. Durante la pandemia actual es perentorio disponer de resultados rápidos y confiables para la toma de decisiones consensuada. Detectar las barreras y los diferentes aspectos de mejora determina una auditoría interna con el objetivo de una mejora continua. Los trabajos colaborativos permiten establecer redes entre diferentes profesionales con el objetivo final de mejorar la calidad de la gestión, las políticas sanitarias y la atención a los pacientes en tiempos de constante cambio.

\section{Financiamiento}

Los autores declaran no haber recibido fuente de financiamiento para la confección de este artículo. 


\section{Conflicto de intereses}

Los autores declaran no tener conflictos de interés en esta publicación.

\section{Responsabilidades éticas}

Protección de personas y animales. Los autores declaran que para esta investigación no se han realizado experimentos en seres humanos ni en animales.

Confidencialidad de los datos. Los autores declaran que han seguido los protocolos de su centro de trabajo sobre la publicación de datos de pacientes.
Derecho a la privacidad y consentimiento informado. Los autores declaran que en este artículo no aparecen datos de pacientes.

\section{Bibliografía}

1. Moher D, Glasziou P, Chalmers I, Nasser M, Bossuyt PMM, Korevaar DA, et al. Increasing value and reducing waste in biomedical research: who's listening? Lancet. 2016;387:1573-86.

2. Harris PA, Taylor R, Minor BL, Elliott V, Fernandez M, O'Neal L, et al. The REDCap consortium: Building an international community of software platform partners. J Biomed Inform. 2019;95:103208.

3. COVIDSurg Collaborative. Delaying surgery for patients with a previous SARS-CoV-2 infection. Br J Surg. 2020;107:e601-2.

4. Pérez C, Picciochi M, Martín J, Calvache JA. Global Perioperative Surgery Research. A call to Latin America. Colomb J Anesthesiol. 2020;49:e942. 